\title{
ABUNDANCIA RELATIVA Y PATRONES DE ACTIVIDAD DE LOS MAMÍFEROS DE LOS CHIMALAPAS, OAXACA, MÉXICO
}

\author{
IVÁN LIRA-TORRES ${ }^{1} \&$ MIGUEL BRIONES-SALAS ${ }^{2}$ \\ ${ }^{1}$ Universidad del Valle de México, Campus Coyoacán, Escuela de Ciencias de la Salud, Escuela de \\ Medicina Veterinaria y Zootecnia. Calzada de Tlalpan No. 3058, Col. Santa Ursula Coapa, Delegación \\ Coyoacán, México D.F. \\ <ilira_12@hotmail.com> \\ ${ }^{2}$ Centro Interdisciplinario de Investigación para el Desarrollo Integral Regional, Unidad Oaxaca \\ (CIIDIR-OAX.), IPN. Hornos 1003, Santa Cruz Xoxocotlán, Oaxaca, Oax., C.P.71230; <mbriones@, \\ ipn.mx>
}

Lira-Torres, I. \& Briones-Salas, M. 2012. Abundancia relativa y patrones de actividad de los mamíferos de los Chimalapas, Oaxaca, México. Acta Zoológica Mexicana (n. s.), 28(3): 566-585.

RESUMEN. La selva de los Chimalapas al sureste de México, es una de las regiones prioritarias para la conservación. En esta región existen áreas de bosques y selvas en buen estado de conservación que no han sido exploradas anteriormente. El estudio de las poblaciones de mamíferos así como los análisis de abundancia relativa y patrones de actividad pueden ayudar a entender la estructura del ecosistema de esta región. Por medio del uso de cámaras trampa, se determinó la abundancia relativa así como los patrones de actividad de los mamíferos medianos y grandes en la región de los Chimalapas, en Oaxaca, México. Se realizaron dos periodos de muestreo fotográfico en la temporada seca de 2009 y la lluviosa de 2010 con un total de 54 cámaras trampas en cada periodo. Con un esfuerzo total de muestreo de 4,860 días-trampa, se lograron registrar 22 especies, 20 de mamíferos medianos y grandes y dos de aves. El $50 \%$ de las especies se encuentran dentro de alguna categoría de riesgo por las leyes nacionales e internacionales. Las especies más abundantes fueron Cuniculus paca, Tayassu pecari, Dasyprocta mexicana, Pecari tajacu, Tapirus bairdii y Dasypus novemcinctus, mientras que la menos abundante fue Urocyon cinereoargenteus. Se registraron tres tipos de patrones de actividad: Nocturno-Crepuscular; con 13 especies, Diurnos-Nocturno-Crepuscular (24 hr); tres especies, y Diurnos con cinco especies. Con esta información se pretende contribuir a la planeación del manejo y conservación de los mamíferos en esta importante región de México.

Palabras clave: Bosque tropical perennifolio, cámaras trampa, Santa María Chimalapa, mamíferos medianos y grandes, patrones de actividad.

Lira-Torres, I. \& Briones-Salas, M. 2012. Relative abundance, and activity patterns of mammals at Chimalapa's forest, Oaxaca, Mexico. Acta Zoológica Mexicana (n. s.), 28(3): 566-585.

ABSTRACT. The Chimalapa's Forest at the southeast of Mexico is one of the priority regions for conservation. There are preserved areas of tropical forests that have not been explored previously. The

Recibido: 02/05/2012; aceptado: 20/09/2012. 
study of populations of mammals that live in this region, as well as the analysis of relative abundance and activity patterns can help us to understand the functioning of the ecosystem. Using the technique of camera- traps, we determine the relative abundance and activity patterns of medium and large mammals at Chimalapa's Forest, Oaxaca. There were two photographic sampling periods: the 2009 dry season and the 2010 rainy season using a total of 54 camera- traps in each period. The total effort was 4,860 trapdays; we recorded 22 species, 20 medium and large mammals, and two birds. The $50 \%$ of the species are included in a category of endangered by national and international laws. The most abundant species were Cuniculus paca, Tayassu pecari, Dasyprocta mexicana, Pecari tajacu, Tapirus bairdii and Dasypus novemcinctus, whereas the less abundant was Urocyon cinereoargenteus. There were three types of patterns of activity: Nocturnal-twilight; with 13 species, diurnal-nocturnal-twilight ( $24 \mathrm{hrs}$ ); three species, and five diurnal species. Our results are a contribution to the planning of the management and the conservation of the mammals at this important region of Mexico.

Key words: Activity schedules, camera traps, Santa Maria Chimalapa, medium and large mammals, tropical rain forest.

\section{INTRODUCCIÓN}

La Selva Zoque que incluye la Sierra Atravesada (región de los Chimalapas) en Oaxaca hasta la Sierra Madre de Chiapas, es una de las regiones menos conocidas biológicamente del Sureste de México; sin embargo, los pocos estudios realizados han demostrado que la zona es muy rica en biodiversidad. Para el caso de los mamíferos se han registrado a la fecha 149 especies que representan el $76 \%$ con respecto al total registrado en Oaxaca (Briones-Salas \& Sánchez-Cordero; 2004, Olguín Monroy et al. 2008, Lira et al. 2012); de éstas, algunas especies se encuentran en peligro de extinción como el jaguar (Panthera onca), el tapir (Tapirus bairdii), el pecarí de labios blancos (Tayassu pecari) y el mono araña (Ateles geoffroyi) (Lira et al. 2012). La fauna de esta región es poco conocida debido principalmente a su intrincada topografía que ocasiona que los estudios sean difíciles de realizar y, aunque hay una gran presencia de especies, las observaciones directas de los mismos son relativamente escasas (Arriaga et al. 2000, Galindo-Leal \& Lira 2011a).

La abundancia y densidad son atributos de la población que varían con el tiempo y en el espacio y son de gran importancia para los estudios de manejo y conservación de fauna silvestre, ya que permiten comparar poblaciones, dar seguimiento a variaciones temporales o a la dinámica poblacional y evaluar de forma indirecta la calidad de los hábitats (Wilson et al. 1996, Walker et al. 2000). Ya que la información para calcular la densidad de los mamíferos neotropicales es costosa y difícil de obtener por varias razones (hábitos nocturnos, bajo número de individuos y evasividad), se recomienda el uso de índices de abundancia relativa (Sutherland 1996). Estos índices son fáciles de estimar y se expresan como el número de individuos por unidad de esfuerzo (Maffei et al. 2002, Yasuda 2004, Rovero \& Marshall 2009, Lira-Torres \& Briones-Salas 2011, Monroy-Vilchis et al. 2011).

A principios de la década de los 80 se comenzaron a utilizar las cámaras trampa y se han convertido en una herramienta importante para el monitoreo de especies, 
con bajas densidades y difíciles de observar, cubriendo además un amplio rango de ambientes que otros métodos no permiten (Silveira et al. 2003, Pinto de Sá Alves \& Andriolo 2005, Srbek-Araujo \& García 2005, Monroy-Vilchis et al. 2009). Esta herramienta se usa para múltiples propósitos, desde identificar especies de manera individual, hasta evaluar el tamaño de poblaciones. Estudios recientes con el uso sistematizado de cámaras trampa, han ayudado a estimar la densidad de la población, describir patrones de actividad (Maffei et al. 2002, Rumiz et al. 2002, Pinto de Sá Alvez \& Andriolo 2005, Monroy-Vilchis et al. 2009), identificar el uso del hábitat (Bowkett et al. 2007, Lira-Torres \& Briones-Salas 2011, Monroy-Vilchis et al. 2009), así como describir el comportamiento de algunas especies (Maffei et al. 2002, 2005, Trolle \& Kéry 2003, 2005, Dillon 2005, Di Bitetti et al. 2006, Haines et al. 2006). Todo lo anterior, convierte el uso de las cámaras trampa en uno de las más importantes y versátiles métodos para estudios de investigación biológica con fines de conservación (Krausman 2002, Maffei et al. 2002, Trolle \& Kéry 2003, Wallace et al. 2003, Maffei et al. 2004, Di Bitetti et al. 2006, Soisalo \& Cavalcanti 2006, Dillon \& Kelly 2008, Maffei \& Noss 2008, Trolle 2008).

Así mismo, el cálculo de la frecuencia de captura mediante el empleo de las cámaras trampa, se ha utilizado como un índice de abundancia relativa (IAR) mostrando una correlación con las densidades (Morruzzi et al. 2002, Maffei et al. 2002, Silveira et al. 2003, De Almeida et al. 2004, Yasuda 2004, Rovero \& Marshall 2009, Jenks et al. 2011, Lira-Torres \& Briones-Salas 2011, Monroy-Vilchis et al. 2011).

Por lo anterior, los objetivos de este trabajo son determinar la abundancia relativa y patrones de actividad de los mamíferos medianos y grandes de la región de los Chimalapas, Oaxaca, México, mediante la técnica de cámaras trampa.

\section{MATERIALES Y MÉTODOS}

Área de estudio. El trabajo de campo se realizó dentro de los terrenos comunales de la Fortaleza, municipio de Santa María Chimalapa. Se ubica al sureste del estado de

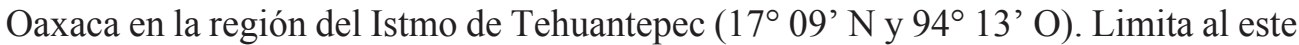
con la Sierra del Espinazo del Diablo, al sur con la Sierra Atravesada, al norte con Los Uxpanapas, Veracruz y al oeste con el río Oaxaca (Arriaga et al. 2000). El clima predominante es cálido húmedo con lluvias en verano; Am(f) y (A)C(w2) (García 1973). La precipitación y temperatura varían de 3000 a $3500 \mathrm{~mm}$ y de 18 a $22^{\circ} \mathrm{C}$, respectivamente (Wendt 1989, Arriaga et al. 2000). Los tipos de vegetación dominantes son el bosque tropical perennifolio y subperennifolio, vegetación secundaria y pastizales introducidos (Rzedowski 1991, González 2004, Torres Colín 2004). Los suelos son de tipo eútrico (Alfaro 2004). El sistema hidrográfico es alimentado por las vertientes septentrionales de los ríos Oaxaca, Uxpanapa, y del escurrimiento de la zona central del río Coatzacoalcos (Ortiz Pérez et al. 2004) (Fig. 1). 


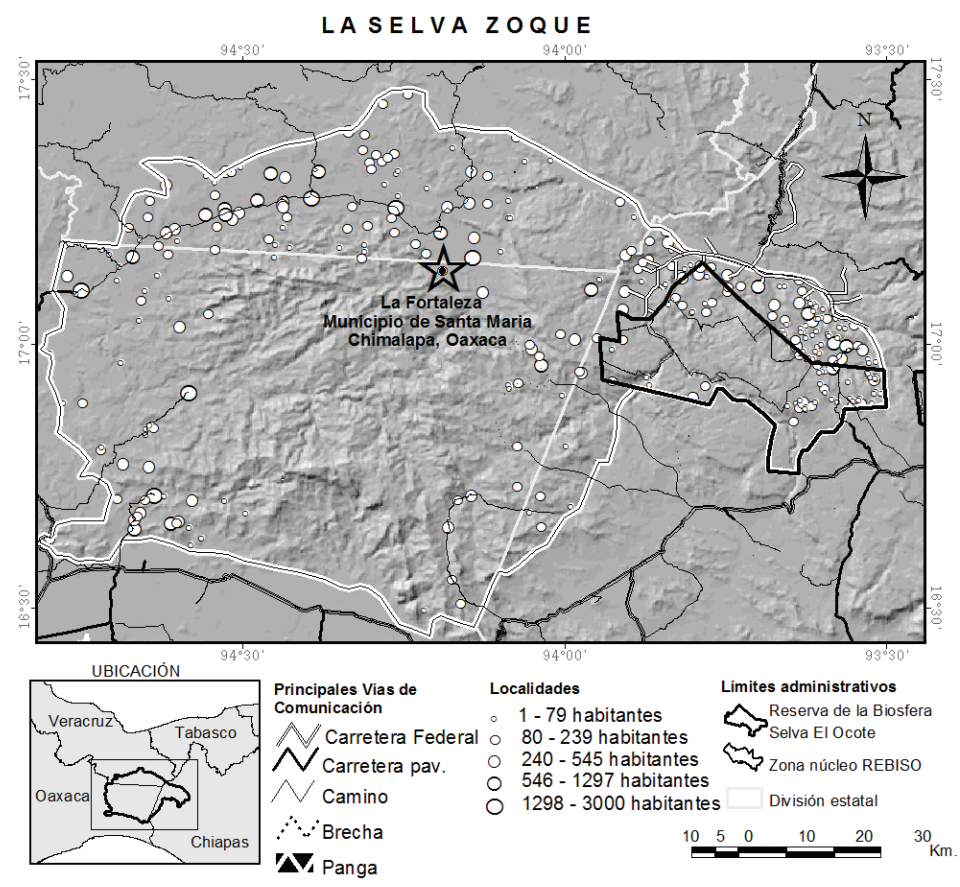

Figura 1. Ubicación geográfica del área de estudio dentro de la región de los Chimalapas, Oaxaca, México.

Se realizaron dos periodos de muestreo fotográfico. El primero se llevó a cabo durante la temporada seca del 2009; del 13 de mayo al 23 de julio y se colocaron 54 cámaras trampa durante 60 días de muestreo efectivos. El segundo muestreo se realizó durante la temporada lluviosa del 2010, del 24 de julio al 24 de agosto, colocando el mismo número de cámaras trampa, pero debido a las fuertes lluvias, los días de muestreo efectivo se redujeron a 30 . El diseño establecido para el muestreo es resultado de la mesa de trabajo del primer Censo Nacional del Jaguar (Panthera onca) y sus presas (CENJAGUAR) (Chávez et al. 2007), en donde se acordó abarcar una superficie de $80 \mathrm{~km}^{2}$ para los sitios prioritarios para la conservación de esta especie y con altas densidades, como la Selva Zoque en Oaxaca (Medellín et al. 2006; Lira \& Ramos-Fernández 2007).

Las estaciones de muestreo se instalaron en veredas naturales, bordes de montañas, cañadas, arroyos secos y márgenes de río, espaciadas una de otra a una distancia de 1.5 a $3 \mathrm{~km}$ para no dejar grandes vacíos sin muestrear. En cada estación de muestreo se instaló una cámara trampa modelo Cuddeback Digital ${ }^{\circledR}$, a una altura no mayor de 40 o $50 \mathrm{~cm}$ del nivel del suelo, dependiendo de la topografía e inclinación del área de muestreo. El circuito de estas cámaras fue programado para permanecer 
activo durante las 24 horas. La posición de cada una de ellas fue georeferenciada con un geoposicionador modelo Garmin etrex ${ }^{\circledR}$. Posteriormente, se revisaron una vez al mes, en cada fotografía detectada se imprimió la hora y la fecha. El esfuerzo total de muestreo se obtuvo multiplicando el número total de cámaras por el total de días de muestreo (Medellín et al. 2006).

Los registros fotográficos obtenidos se prepararon de acuerdo a la propuesta de Botello et al. (2004, 2007), el cual permite consultar las fotografías digitales en cualquier computadora con software de fácil acceso. La organización propone que el nombre del archivo lleve la letra inicial del género, las tres primeras letras del nombre específico, seguido de la clave del país, entidad y municipio. Por último, las iniciales del primer nombre y apellido del colector, así como el número de fotocolecta y el tipo de archivo.

Posteriormente las fotografías fueron depositadas en la Colección de Mamíferos (OAX-MA.026.0497) del Centro Interdisciplinario de Investigación para el Desarrollo Integral Regional, Unidad Oaxaca (CIIDIR-OAX.), del Instituto Politécnico Nacional (IPN). Los individuos de las especies fotografiadas fueron identificados con base en literatura especializada (Hall 1981; Reid 1997; Aranda 2000; Ceballos \& Oliva 2005). La clasificación y nomenclatura se basó en la actualización sistemática y taxonómica propuesta por Ceballos, Arroyo-Cabrales y Medellín (Ceballos \& Oliva 2005) y Bello \& Reyna-Hurtado (2010).

Para obtener el índice de abundancia relativa (IAR) de cada especie, se utilizó una fórmula probada por otras autoridades (Maffei et al. 2002, Sanderson 2004; Azuara 2005; Jenks et al. 2011):

$$
\mathrm{IAR}=\mathrm{C} / \mathrm{EM} * 1000 \text { días-trampa }
$$

Donde: $\mathrm{C}=$ Capturas o eventos fotografiados.

$\mathrm{EM}=$ Esfuerzo de Muestreo (No. de cámaras * días de monitoreo) Estacional o Total.

1000 días-trampa (Unidad Estándar).

Se consideraron como registros fotográficos independientes sólo los siguientes casos: a) fotografías consecutivas de diferentes individuos, b) fotografías consecutivas de la misma especie separadas por 24 horas. Este criterio fue aplicado cuando no era claro si una serie de fotografías correspondían al mismo individuo, de modo que las fotografías tomadas antes de 24 horas se consideraron como un solo registro, c) fotografías no consecutivas de la misma especie (Medellín et al. 2006; Lira-Torres \& Briones-Salas 2011, Monroy-Vilchis et al. 2011).

El patrón de actividad se determinó para aquellas especies de las que se obtuvieron al menos 11 registros fotográficos independientes con la hora visible, (Maffei et al. 2002, Monroy-Vilchis et al. 2009). Los registros obtenidos se ordenaron por 
intervalos de dos horas (Monroy-Vilchis et al. 2011). Los patrones de actividad se agruparon en tres unidades: a) diurnos, cuando en las fotografías se observaba luz solar; b) nocturnos cuando no había luz solar, y c) crepusculares, cuando se obtuvieron al amanecer (06:00-08:00hr) o al atardecer (18:00-20:00hr) (Monroy-Vilchis et al. 2011).

\section{RESULTADOS}

Durante los dos periodos de muestreo se registraron 22 especies, 20 de la clase Mammalia y dos de la de Aves. El 50\% del total de especies registradas se encuentran en alguna categoría de riesgo de acuerdo con las normas mexicanas (SEMARNAT 2010): $n=10 ; 8$ mamíferos y 2 aves; y el $25 \%$ dentro de alguna categoría de riesgo por normas internacionales (IUCN 2011: $n=5$; 4 mamíferos y 1 ave (Cuadro 1).

Durante el primer muestreo fotográfico (2009) con un esfuerzo de 3,240 días/ trampa se obtuvieron 868 fotografías, 588 independientes. Se registraron 22 especies de las dos clases antes mencionadas, que corresponden a 21 géneros y 16 familias. Los mamíferos estuvieron representados por 20 especies, mientras que las aves solamente por dos.

En el segundo muestreo (2010) con la mitad del esfuerzo, 1,620 días/trampa, se obtuvieron 74 fotografías, 72 fueron independientes. Se registraron 11 especies, correspondientes a 11 géneros y 10 familias de las dos clases, 10 especies de mamíferos y sólo una de aves. El esfuerzo total de muestreo en los dos años fue de 4,860 días/ trampa (Cuadro 1).

Abundancia. Las especies más abundantes en las estaciones de trampeo para ambos periodos (2009 - 2010) fueron Cuniculus paca (IAR $=26.95, n=131), T a$ yassu pecari $(\mathrm{IAR}=25.10, n=122)$, Dasyprocta mexicana $(\mathrm{IAR}=20.37, n=99)$, Pecari tajacu (IAR $=17.48, n=85)$, Tapirus bairdii (IAR $=8.23, n=40)$ y Dasypus novemcinctus (IAR $=5.76, n=28$ ), mientras que la menos abundante fue Urocyon cinereoargenteus (IAR $=0.20, n=1$ ). Para el caso de las aves, Crax rubra fue más abundante (IAR $=10.90, n=53)$ que Penelope purpurascens $(\mathrm{IAR}=0.41, n=2)$.

Durante la temporada seca (2009), las especies más abundantes fueron Cuniculus paca $(\mathrm{IAR}=37.96, n=123)$ y Tayassu pecari $(\mathrm{IAR}=37.65, n=122)$, mientras que las especies menos abundantes fueron Urocyon cinereoargenteus $(\mathrm{IAR}=0.30, n=1)$ y Procyon lotor $(\mathrm{IAR}=0.30, n=1)$. Para la temporada lluviosa (2010) se observaron variaciones, las especies más abundantes fueron Pecari tajacu $(\mathrm{IAR}=14.81, n=24)$ y Tapirus bairdii (IAR $=11.11, n=18$ ), mientras que las menos abundante fueron Leopardus pardalis, Eira barbara y Nasua narica (IAR $=0.61, n=1$ ) (Fig. 2, Cuadro 2). Los órdenes con valores más altos de IAR en orden descendiente fueron Rodentia $(35.45 \%)$, Artiodactyla (33.63\%), Carnivora (9.54\%), Perissodactyla $(6.06 \%)$ y Cingulata (4.24\%) (Cuadro 3). 
Cuadro 1. Especies registradas por cámaras trampa en la región de los Chimalapas, Oaxaca, México.

\begin{tabular}{|c|c|c|c|c|c|c|}
\hline Familia & Especie & Nombre Común & $\begin{array}{c}2009 \\
\text { (Secas) }\end{array}$ & $\begin{array}{c}2010 \\
\text { (Lluvias) }\end{array}$ & $\begin{array}{c}\text { Nom-059 } \\
2010 \\
\end{array}$ & UICN \\
\hline Caluromyidae & $\begin{array}{l}\text { Caluromys } \\
\text { derbianus }\end{array}$ & Tlacuache dorado & $\mathrm{X}$ & - & A & - \\
\hline \multirow[t]{2}{*}{ Didelphidae } & $\begin{array}{l}\text { Didelphis } \\
\text { marsupialis }\end{array}$ & Tlacuache común & $\mathrm{X}$ & - & - & - \\
\hline & $\begin{array}{l}\text { Philander } \\
\text { oposum }\end{array}$ & $\begin{array}{l}\text { Tlacuache cuatro } \\
\text { ojos }\end{array}$ & $\mathrm{X}$ & - & - & - \\
\hline Dasypodidae & $\begin{array}{l}\text { Dasypus } \\
\text { novemcinctus }\end{array}$ & $\begin{array}{l}\text { Armadillo nueve } \\
\text { bandas }\end{array}$ & $\mathrm{X}$ & $\mathrm{X}$ & - & - \\
\hline Canidae & $\begin{array}{l}\text { Urocyon } \\
\text { cinereoargenteus }\end{array}$ & Zorra gris & $\mathrm{X}$ & - & - & - \\
\hline \multirow[t]{3}{*}{ Felidae } & $\begin{array}{l}\text { Puma } \\
\text { yagouaroundi }\end{array}$ & Jaguarundi & $\mathrm{X}$ & - & A & - \\
\hline & $\begin{array}{l}\text { Leopardus } \\
\text { pardalis }\end{array}$ & Ocelote & $\mathrm{X}$ & $\mathrm{X}$ & $\mathrm{P}$ & - \\
\hline & Leopardus wiedii & Tigrillo & $\mathrm{X}$ & - & $\mathrm{P}$ & NT \\
\hline Mustelidae & Eira barbara & Viejo de monte & $\mathrm{X}$ & $X$ & $\mathrm{P}$ & - \\
\hline Mephitidae & $\begin{array}{l}\text { Conepatus } \\
\text { semistriatus }\end{array}$ & Zorrillo tropical & $\mathrm{X}$ & - & $\operatorname{Pr}$ & - \\
\hline \multirow[t]{2}{*}{ Procyonidae } & Nasua narica & Tejón & $\mathrm{X}$ & $\mathrm{X}$ & - & - \\
\hline & Procyon lotor & Mapache & $\mathrm{X}$ & $\mathrm{X}$ & - & - \\
\hline Tapiridae & Tapirus bairdii & Tapir & $\mathrm{X}$ & $\mathrm{X}$ & $\mathrm{P}$ & E \\
\hline Cervidae & Mazama temama & Temazate & $\mathrm{X}$ & $\mathrm{X}$ & - & - \\
\hline \multirow[t]{2}{*}{ Tayassuidae } & Tayassu pecari & Marín & $\mathrm{X}$ & - & $\mathrm{P}$ & NT \\
\hline & Pecari tajacu & Pecarí de collar & $\mathrm{X}$ & $\mathrm{X}$ & - & - \\
\hline Sciuridae & $\begin{array}{l}\text { Sciurus } \\
\text { aureogaster }\end{array}$ & Ardilla gris & $\mathrm{X}$ & - & - & - \\
\hline Cuniculidae & Cuniculus paca & Tepezcuintle & $\mathrm{X}$ & $\mathrm{X}$ & - & - \\
\hline Dasyproctidae & $\begin{array}{l}\text { Dasyprocta } \\
\text { mexicana }\end{array}$ & Guaqueque negro & $\mathrm{X}$ & $\mathrm{X}$ & - & $\mathrm{CE}$ \\
\hline Leporidae & $\begin{array}{l}\text { Sylvilagus } \\
\text { floridanus }\end{array}$ & Conejo & $\mathrm{X}$ & - & - & - \\
\hline \multirow[t]{2}{*}{ Cracidae } & Crax rubra & Hocofaisán & $X$ & $\mathrm{X}$ & $\mathrm{A}$ & $\mathrm{V}$ \\
\hline & $\begin{array}{l}\text { Penelope } \\
\text { purpurascens }\end{array}$ & Cojolita & $X$ & - & A & - \\
\hline
\end{tabular}

Nom-059-2010: $\mathrm{A}=$ Amenazadas, $\mathrm{P}=$ En peligro de extinción, $\mathrm{Pr}=$ Sujetas a protección especial. $\mathrm{UICN}$ : NT = Casi amenazada, $\mathrm{E}=$ En Peligro, $\mathrm{CE}=$ Críticamente en Peligro, $\mathrm{V}=$ Vulnerable. 


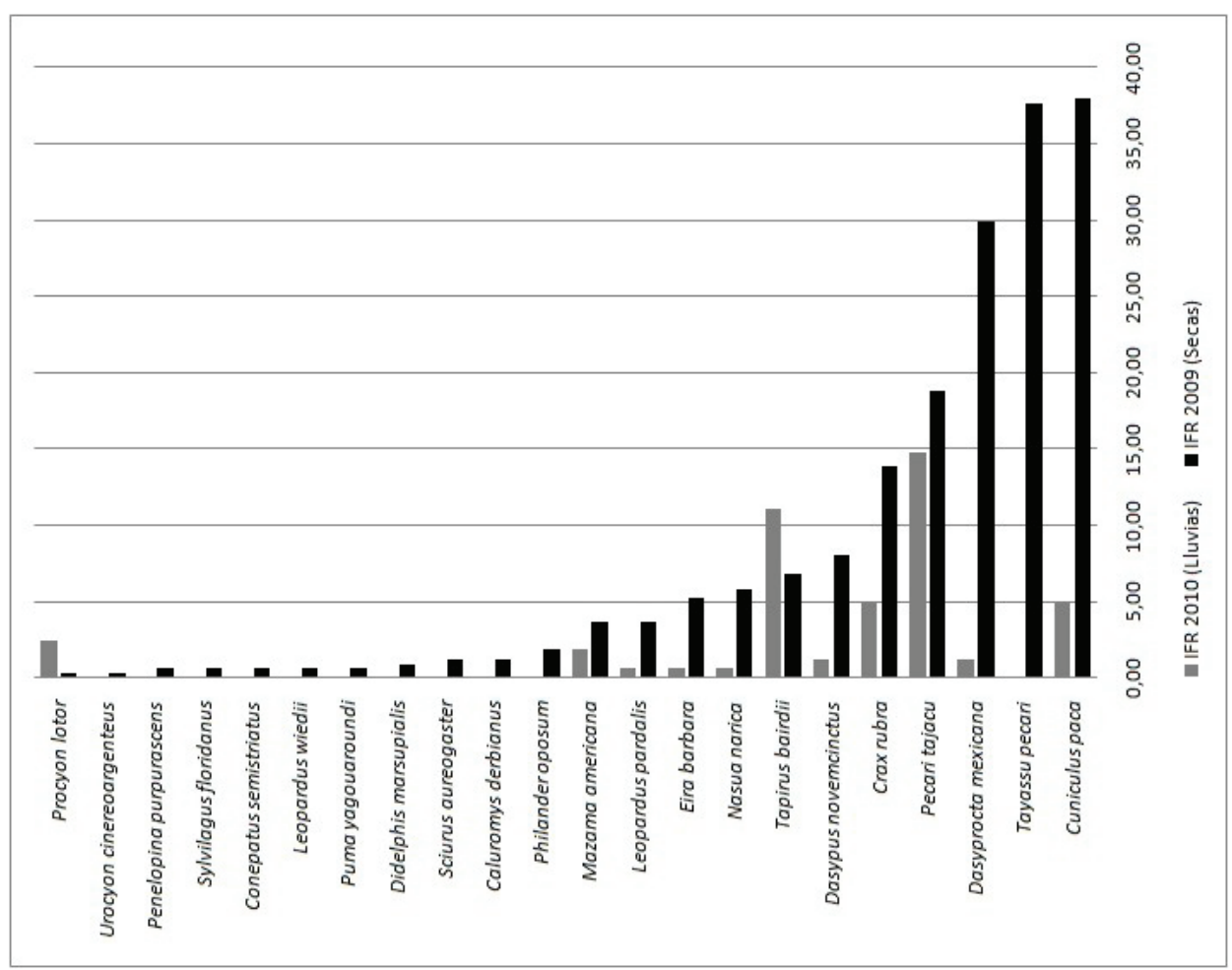

Figura 2. Índices de abundancia relativa (IAR) de las especies presentes en la región de los Chimalapas, Oaxaca, México.

Patrones de Actividad. Se registraron todos los tipos de patrones de actividad (Cuadro 4): a) Nocturno-Crepuscular, el 63\% de las especies se registró dentro de este patrón, con actividad entre las 19:00 y 00:00h y entre las 00:01 y 05:00h; b) Diurnos-Nocturnos-Crepusculares (Actividad durante las 24 horas); y c) Diurnos: con periodos entre las 05:00-18:00h, incluyendo a las aves.

\section{DISCUSIÓN}

De las 149 especies de mamíferos previamente registradas en la Selva Zoque (Lira et al. 2012) y las 29 registradas para la región de los Chimalapas (Olguin Monroy et al. 2008), este trabajo registró por medio de la técnica de cámaras trampa, 22 especies; es decir el $66.66 \%$ y el $75.86 \%$ respectivamente. Cabe mencionar que en ambos estudios, se utilizaron técnicas de colecta directa, métodos indirectos mediante el registro de huellas, excretas e individuos, empleando recorridos en transectos lineales (Lira et al. 2004, Lira 2006, Tobler et al. 2009, Roberts et al. 2006). 


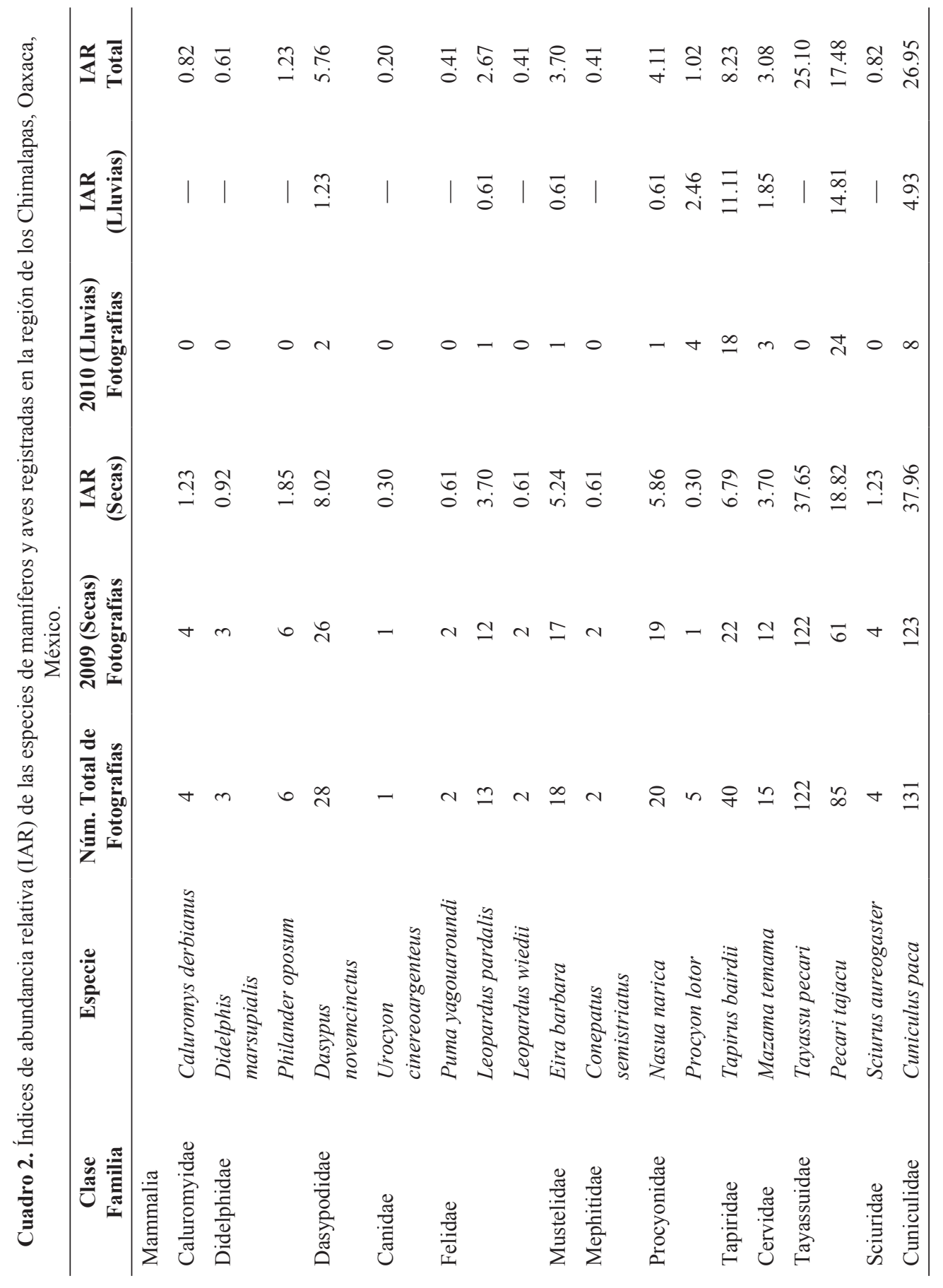




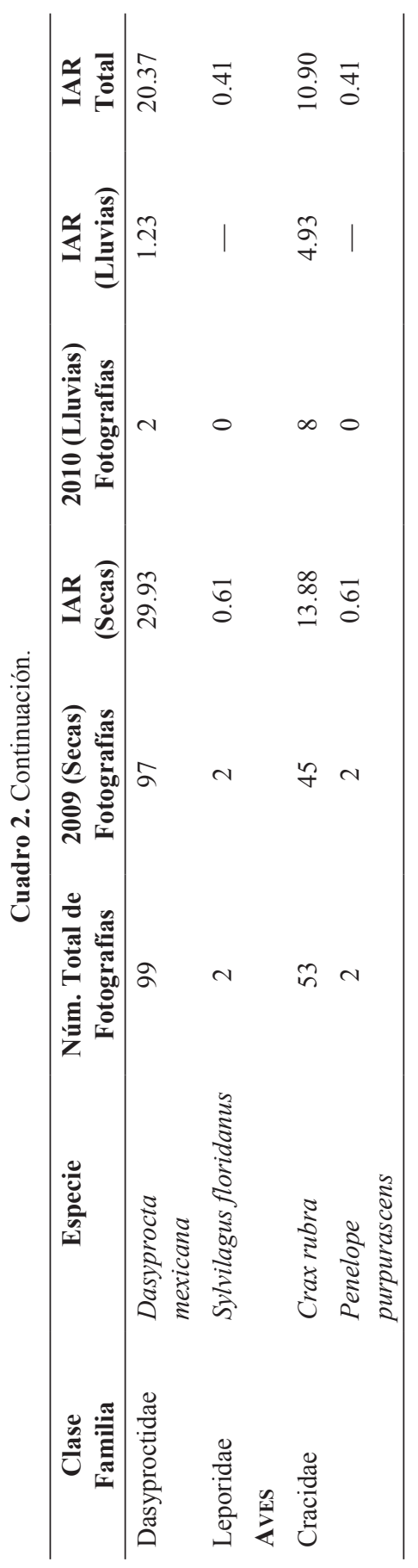


Cuadro 3. Índices de abundancia relativa (IAR) de los órdenes registrados en la región de los Chimalapas, Oaxaca, México.

\begin{tabular}{clcccccc}
\hline Clase & \multicolumn{1}{c}{ Órdenes } & $\begin{array}{c}\text { Núm. } \\
\text { Total de } \\
\text { Fotografías }\end{array}$ & $\begin{array}{c}\text { 2009 } \\
\text { (Secas) } \\
\text { Fotografías }\end{array}$ & $\begin{array}{c}\text { IAR } \\
\text { Fo10 } \\
\text { Fotografías }\end{array}$ & IAR & $\begin{array}{c}\text { IAR } \\
\text { Total }\end{array}$ \\
\hline Mammalia & Didelphimorphia & 13 & 13 & 4.01 & - & - & 2.67 \\
& Cingulata & 28 & 26 & 8.02 & 2 & 1.23 & 5.76 \\
& Carnivora & 63 & 56 & 17.28 & 7 & 4.32 & 12.96 \\
& Perissodactyla & 40 & 22 & 6.79 & 18 & 11.11 & 8.23 \\
& Artiodactyla & 222 & 195 & 60.18 & 27 & 16.66 & 45.67 \\
& Rodentia & 234 & 224 & 69.13 & 10 & 6.17 & 48.14 \\
& Lagomorpha & 2 & 2 & 0.61 & - & - & 0.41 \\
& Gallifornes & 55 & 47 & 14.50 & 8 & 4.93 & 11.31 \\
\hline
\end{tabular}

Esto sin duda demuestra la efectividad de esta técnica, por lo que el uso de cámaras trampa es una alternativa excelente que complementa los métodos convencionales. Además habrá que tomar en cuenta que este trabajo es uno de los pocos realizados en la región de los Chimalapas, Oaxaca (Selva Zoque), zona por demás inaccesible, accidentada y escarpada del sureste de México.

Con este estudio, la mayoría de los mamíferos tropicales de tamaño mediano y grande del sureste de México fueron registrados, sin embargo otras especies como Odocoileus virginianus y Canis latrans, especies comunes en hábitats trasformados del norte, centro y sur de México, han sido reconocidas en áreas limítrofes por evidencias indirectas de pieles y cráneos, evidenciando su escasa presencia en la zona norte del área de estudio (Lira et al. 2012).

La región del Istmo de Tehuantepec, sitio donde se ubican los Chimalapas se caracteriza por presentar variaciones climatológicas en intervalos de varios años. Durante el segundo año del muestreo (2010), se registró una de las más altas precipitaciones pluviales en los últimos diez años. Esto sin duda, ocasionó diferencias en cuanto al número de registros fotográficos efectivos obtenidos en los dos años; 2009 (588 fotos en la temporada de secas) vs 2010 (72 registros fotográficos en la temporada de lluvia). Este exceso de humedad durante el segundo año afectó el funcionamiento de las cámaras trampa, lo que limitó su sensibilidad de barrido o rastreo. A pesar de esos inconvenientes, se obtuvo información de especies de mamíferos medianos y grandes y de crácidos, especies apreciadas por las comunidades marginadas por constituir una fuente de proteína animal (Galindo-Leal \& Lira 2011b).

Además, con ésta técnica fue posible obtener información sobre aspectos ecológicos como abundancia relativa, patrones de actividad e incluso, información sobre aspectos de la reproducción, como registros de apareamientos de Tayassu pecari y 
Cuadro 4. Patrones de actividad de las especies de mamíferos y aves más abundantes en la región de los Chimalapas, Oaxaca, México.

\begin{tabular}{|c|c|c|c|c|c|}
\hline Clase & Familia & Especie & Registros & Horarios & $\begin{array}{c}\text { Patrones de } \\
\text { actividad }\end{array}$ \\
\hline \multirow[t]{20}{*}{ Mammalia } & Caluromyidae & Caluromys derbianus & 4 & $\begin{array}{l}19: 00-22: 00 \mathrm{~h} \\
01: 00-04: 00 \mathrm{~h}\end{array}$ & $\begin{array}{l}\text { Nocturno/ } \\
\text { Crepuscular }\end{array}$ \\
\hline & Didelphidae & Didelphis marsupialis & 3 & $\begin{array}{l}20: 00-23: 00 \mathrm{~h} \\
01: 00-02: 00 \mathrm{~h}\end{array}$ & $\begin{array}{l}\text { Nocturno/ } \\
\text { Crepuscular }\end{array}$ \\
\hline & & Philander oposum & 6 & 00:00-05:00 h & $\begin{array}{l}\text { Nocturno/ } \\
\text { Crepuscular }\end{array}$ \\
\hline & Dasypodidae & Dasypus novemcinctus & 28 & $20: 00-05: 00 \mathrm{~h}$ & $\begin{array}{l}\text { Nocturno/ } \\
\text { Crepuscular }\end{array}$ \\
\hline & Canidae & Urocyon cinereoargenteus & 1 & $20: 00-21: 00 \mathrm{~h}$ & Nocturno \\
\hline & Felidae & Puma yagouaroundi & 2 & $\begin{array}{l}09: 00-10: 00 \mathrm{~h} \\
17: 00-18: 00 \mathrm{~h}\end{array}$ & Diurno \\
\hline & & Leopardus pardalis & 13 & $19: 00-05: 00 \mathrm{~h}$ & $\begin{array}{l}\text { Nocturno/ } \\
\text { Crepuscular }\end{array}$ \\
\hline & & Leopardus wiedii & 2 & $21: 00-23: 00 \mathrm{~h}$ & Nocturno \\
\hline & Mustelidae & Eira barbara & 18 & 07:00-19:00 h & Diurno \\
\hline & Mephitidae & Conepatus semistriatus & 2 & $\begin{array}{l}20: 00-21: 00 \mathrm{~h} \\
00: 00-04: 00 \mathrm{~h}\end{array}$ & $\begin{array}{l}\text { Nocturno/ } \\
\text { Crepuscular }\end{array}$ \\
\hline & Procyonidae & Nasua narica & 20 & $06: 00-17: 00 \mathrm{~h}$ & Diurno \\
\hline & & Procyon lotor & 5 & 04:00-05:00 h & Crepuscular \\
\hline & Tapiridae & Tapirus bairdii & 40 & 20:00-05:00 h & $\begin{array}{l}\text { Nocturno/ } \\
\text { Crepuscular }\end{array}$ \\
\hline & Cervidae & Mazama temama & 15 & $\begin{array}{l}18: 00-01: 00 \mathrm{~h} \\
07: 00-14: 00 \mathrm{~h}\end{array}$ & $\begin{array}{l}\text { Nocturno/ } \\
\text { Diurno }\end{array}$ \\
\hline & Tayassuidae & Tayassu pecari & 122 & $00: 00-23: 00 \mathrm{~h}$ & $\begin{array}{l}\text { Diurno/ } \\
\text { Nocturno/ } \\
\text { Crepuscular }\end{array}$ \\
\hline & & Pecari tajacu & 85 & $00: 00-23: 00 \mathrm{~h}$ & $\begin{array}{l}\text { Diurno/ } \\
\text { Nocturno/ } \\
\text { Crepuscular }\end{array}$ \\
\hline & Sciuridae & Sciurus aureogaster & 4 & 06:00-09:00 h & Diurna \\
\hline & Cuniculidae & Cuniculus paca & 131 & 20:00-05:00 h & $\begin{array}{l}\text { Nocturno/ } \\
\text { Crepuscular }\end{array}$ \\
\hline & Dasyproctidae & Dasyprocta mexicana & 99 & 05:00-19:00 h & Diurno \\
\hline & Leporidae & Sylvilagus floridanus & 2 & 07:00-21:00 h & Nocturno \\
\hline \multirow[t]{2}{*}{ Aves } & Cracidae & Crax rubra & 53 & 04:00-18:00 h & Diurno \\
\hline & & Penelope purpurascens & 2 & 08:00-11:00 h & Diurno \\
\hline
\end{tabular}


Tapirus bairdii, y el registro de hembras con crías, como en el caso de Pecari tajacu, Tapirus bairdii y Nasua narica.

Abundancia. Entre los organismos más abundantes en la zona se registraron al tepezcuintle (Cuniculus paca), el marín o pecarí de labios blancos (Tayassu pecari), el guaqueque negro (Dasyprocta mexicana), el jabalí de collar (Pecari tajacu), el tapir centroamericano (Tapirus bairdii) y al armadillo (Dasypus novemcinctus). Todas estas especies previamente identificadas en la zona a través de registros indirectos y como principales presas de caza por las comunidades rurales (Galindo-Leal \& Lira 2011b, Lira-Torres et al. 2012).

A pesar de que en la zona aún se practica la cacería de subsistencia (Lira-Torres \& Briones Salas 2011), la abundancia relativa de las especies antes mencionadas es bastante alta en relación a las otras especies que componen la comunidad de mamíferos medianos y grandes de la región. Para el caso de los dos grandes roedores: tepezcuintle, C. paca; y el guaqueque, D. mexicana, su presencia puede estar favorecida por la presencia de cuevas y túneles que utilizan como refugios y madrigueras, así como por la alta humedad, abundantes árboles frutales (Ficus sp., Pouteria sapota, etc.) y densa vegetación en la región (Gobierno del Estado de Oaxaca 1990, Aparicio 2001).

El pecarí de labios blancos $T$. pecari y el tapir centroamericano T. bairdii, son dos especies que se encuentran dentro de la categoría de "en peligro de extinción" de acuerdo a las leyes mexicanas (NOM-059, SEMARNAT 2010); sin embargo, sus índices de abundancia fueron relativamente altos. Para el caso de T. pecari, su abundancia se relaciona posiblemente a que su cacería se ha vuelto más difícil al refugiarse en sitios de difícil acceso, en los márgenes de los terrenos comunales, en zonas abruptas $\mathrm{y}$ accidentadas, $\mathrm{y}$ en sitios con narcotráfico constante.

Para el caso de T. bairdii se observaron varios ejemplares, así como una hembra con cría en la zona de estudio. Esta especie se registró como la quinta con mayor abundancia relativa; sin embargo, se observaron variaciones en los IAR durante la temporada de lluvia (2010). Estas variaciones posiblemente estén asociadas a una mayor presencia de áreas inundadas en los remanentes de vegetación secundaria por el desbordamiento de lagunas, arroyos y ríos de la región, dificultando el acceso a los comuneros, ganaderos, ejidatarios y cazadores de la región.

Las razones antes mencionadas posiblemente han permitido que estas y otras especies; como la guacamaya roja (Ara macao), el loro cabeza azul (Amazona farino$s a$ ), el zopilote rey (Sarcoramphus papa), el águila de penacho (Spizaetus ornatos), el mono araña (Ateles geoffroyi), el mono aullador (Alouatta palliata), y la nutria de río (Lontra longicaudis), aún sean abundantes en esta región (Lira-Torres et al. 2012).

Los IAR para $P$. tajacu eran de esperarse, ya que es una especie con gran capacidad de adaptación a diferentes tipos de hábitats, así como a presiones de cacería como las registradas por Lira-Torres et al. (2012) para esta región y como lo discuten Fragoso (1988), March (1990) y Bodmer \& Sowls (1996). 
D. novemcinctus fue la sexta especie con alto valor de abundancia relativa. Algunos autores han observado que el fototrampeo no es una técnica adecuada para analizar su abundancia (Weckel et al. 2006, Harmsen et al. 2010, Monroy-Vilchis et al. 2011); sin embargo, en este trabajo se registraron valores de abundancia altos, debidos posiblemente a que muchas de las cámaras trampa fueron colocadas a alturas menores a $40 \mathrm{~cm}$ lo que incrementa la posibilidad de registrar especies de tamaño pequeño como los mismos armadillos, marsupiales y roedores entre otros.

Por otro lado, para el mapache Procyon lotor y la zorra gris Urocyon cinereoargenteus, los índices de abundancia relativa fueron bajos, posiblemente sus poblaciones se encuentren reducidas en esta zona; resultados contrarios a los de Pina et al. (2004), quienes reportan las mismas especies pero abundantes en Sonora, México, con el mismo método de muestreo.

Finalmente, para Penelope purpurascens los IAR muestran que esta especie se encuentra entre las más amenazadas en la selva, a diferencia de Crax rubra en la cual se observa una abundancia más estable. La principal causa de disminución de las poblaciones de estas especies es la pérdida del hábitat que es transformado para actividades humanas como la ganadería y agricultura (Lira-Torres et al. 2012).

Patrones de Actividad. El monitoreo de 24 horas provee datos confiables sobre los patrones de actividad si lo comparamos con otros métodos, como los recorridos a pie en transectos lineales para observaciones directas o censos, aunque no proveen datos contundentes de horarios de actividad. Así mismo, el tamaño corporal de los mamíferos también está relacionado con su patrón de actividad, de manera que los grandes mamíferos del neotrópico que tienen requerimientos energéticos mayores, forrajean durante todo el día, situación observada en las dos especies de pecarís ( $T$. pecari y $P$. tajacu) y el venado temazate (Mazama temama) (Monroy-Vilchis et al. 2011). A diferencia del tapir centroamericano (T. bairdii), cuyos patrones de actividad fueron exclusivamente nocturno/crepusculares, entre 20:00 - 05:00h.

Se ha mencionado (Monroy-Vilchis et al. 2011) que el patrón de actividad de especies de hábitos nocturnos/crepusculares con peso menor de $10 \mathrm{~kg}$ (Caluromys derbianus, Didelphis marsupialis, Philander oposum, Dasypus novemcinctus, Leopardus wiedii, Procyon lotor, Conepatus semistriatus y Sylvilagus floridanus) se relaciona con la evasión del riesgo de depredación. Nuestros resultados contradicen esa afirmación, ya que Eira barbara, Nasua narica, Sciurus aureogaster y Dasyprocta mexicana, fueron activas exclusivamente durante el día, por lo que es probable que factores como la temperatura, disponibilidad de alimento o presas, y nicho tengan mayor influencia sobre su patrón de actividad.

L. pardalis $(\mathrm{n}=13$ fotos), presentó actividad entre las 19:00-00:00 y entre las 00:01-05:00 horas (nocturnos/crepusculares). Este comportamiento, es muy similar a lo encontrado en la isla de Barro Colorado, Panamá, donde se utilizó radio-telemetría y cámaras trampas de forma simultánea (Moreno et al. 2006). Otros autores que uti- 
lizaron radio-telemetría, también reportan que esta especie es activa principalmente durante la noche. Se infiere que esto tiene relación con los patrones de actividad de las principales presas, C. paca, cuyos patrones de actividad coinciden con los de este felino (Ludlow \& Sunquist 1987, Emmons 1988, Crawshaw 1995, Bustamante 2008). En diversos estudios que utilizan cámaras trampa, se ha observado el mismo patrón de comportamiento (Maffei et al. 2005, Di Bitetti et al. 2006, Bustamante 2008). Es evidente que la mayor cantidad de fotografías se obtuvo entre las 19:00 y 00:00h y las 00:01 y 05:00h, esto debido a que con excepción de los primates, la mayoría de los mamíferos neotropicales, como los registrados en los Chimalapas (63\%) son predominantemente nocturnos/crepusculares (Srbek-Araujo \& García 2005).

Con la información obtenida durante este estudio, fue posible conocer la abundancia relativa y los patrones de actividad de algunos de los mamíferos de la región de los Chimalapas, Oaxaca en la Selva Zoque. Entre las especies más amenazadas se encuentran los carnívoros; esta información puede contribuir a la planeación del manejo y conservación de dichas especies. Las estrategias de acción recomendables para continuar con la conservación de los mamíferos en esta área son: 1) Evaluar a mediano y largo plazo el impacto de la cacería, ganadería y utilización del hábitat de los mamíferos en la región; 2) Diseñar e implementar programas de educación ambiental enfocada a la conservación de los mamíferos medianos y grandes del área; 3) Implementar sistemas agrosilvopastoriles y agroforestales, con menor impacto sobre la fauna silvestre, 4) Implementar programas de ecoturismo a nivel regional enfocados a la observación de primates, psitácidos, rapaces, grandes ungulados y cocodrilianos y 5) Establecer un mayor número de Áreas de Conservación Certificadas en la Selva Zoque por parte del gobierno federal, estatal y $\mathrm{ONG}^{\prime}$ 's internacionales.

Agradecimientos. Este trabajo no hubiera sido posible sin el apoyo constante de la Congregación de La Fortaleza, Municipio de Santa María Chimalapa, Oaxaca. Agradecemos al Consejo Nacional de Ciencia y Tecnología (CONACYT), y al PIFI del IPN por las becas otorgadas al primer autor para realizar estudios de Posgrado (registro 239333). Al Laboratorio de Ecología y Conservación de Fauna Silvestre del Instituto de Ecología de la UNAM, Idea Wild (Biodiversity Organization), Fondo Mundial para la Naturaleza (WWF-México), Agencia de Desarrollo Internacional de Estados Unidos (USAID) y el Parque Zoológico de León Guanajuato, por el apoyo logístico brindado, equipo de campo prestado, donado y financiamiento otorgado. MB-S agradece a la SIP (20090672), Comisión de Operación y Fomento a las actividades Académicas (COFFA) y los Estímulos al Desempeño a la Investigación (EDI), del Instituto Politécnico Nacional (IPN) por el apoyo económico recibido, así como al Sistema Nacional de Investigadores (SNI) por su reconocimiento y apoyo.

\section{LITERATURA CITADA}

Alfaro, S. 2004. Suelos, pp. 55-65. In: A. J. García-Mendoza, M. J. Ordóñez \& M. Briones-Salas (Eds.). Biodiversidad de Oaxaca. Instituto de Biología, UNAM, Fondo Oaxaqueño para la Conservación de la Naturaleza-World Wildlife Fund, México. 
Aparicio, R. (Ed.). 2001. Chimalapas. La Última Oportunidad. WWF, SEMARNAP. México.

Aranda, M. 2000. Huellas y otros rastros de los mamíferos grandes y medianos de México. Instituto de Ecología A.C., Xalapa, México.

Arriaga, L., Espinoza, J. M., Aguilar, C., Martínez, E., Gómez, L. \& Loa, E. (coordinadores). 2000. Regiones terrestres prioritarias de México. Comisión Nacional para el Conocimiento y Uso de la Biodiversidad, México.

Azuara, S. D. 2005. Estimación de abundancia de mamíferos terrestres en un área de la Selva Lacandona, Chiapas. Tesis Licenciatura, Facultad de Ciencias, UNAM.

Bello, G. J., \& Reyna-Hurtado, R. 2010. Mazama temama (Kerr 1792), Central America Red Brocket Deer. pp. 166-171. In: Barbanti-Duarte, J. M. \& Gonzalez, S. (Eds.). Neotropical Cervidology: Biology and medicine of Latin American deer. Jaboticabal, Brazil: Funep and Gland, Switzerland: IUCN.

Bodmer, R. E. \& Sowls, K. L. 1996. El Pecarí de Collar, pp. 5-15. In: W. Oliver. (Ed.) Plan de Acción y Evaluación de la Condición actual de los Pecaries. UICN.

Botello, F., Monroy, G., Illoldi-Rangel, P., Trujillo-Bolio, I. \& Sánchez-Cordero, V. 2004. Colección Nacional de Fotocolectas Biológicas (CNFB): Una Propuesta del Uso de la Imagen Digital al Servicio del Conocimiento de la Biodiversidad, pp. 201-207. In: C. Lorenzo, E. Espinoza, M. Briones \& F. A. Cervantes (Eds). Colecciones Mastozoológicas de México. Instituto de Biología-UNAM y Asociación Mexicana de Mastozoología, A.C.

Botello, F., Monroy, G., Illoldi-Rangel, P., Trujillo-Bolio, I. \& Sánchez-Cordero, V. 2007. Sistematización de imágenes obtenidas por fototrampeo: una propuesta de ficha. Revista Mexicana de Biodiversidad, 78: 207- 210.

Bowkett, A., Rovero, F.\& Marshall, A. 2007 The use of camera-trap data to model habitat use by antelope species in the Udzungwa Mountain forests, Tanzania. African Journal of Ecology, 46: 479-487.

Briones-Salas, M. \& Sánchez-Cordero, V. 2004. Mamíferos, pp. 423-447. In: A. J. García Mendoza, M. J. Ordóñez \& M. Briones-Salas (Eds.). Biodiversidad de Oaxaca. Instituto de Biología, Universidad Nacional Autónoma de México, Fondo Oaxaqueño para la Conservación de la Naturaleza, World Wildlife Fund. México.

Bustamante, A. 2008. Densidad y uso de hábitat por los ocelotes (Leopardus pardalis), pumas (Puma concolor) y jaguares (Panthera onca) en la parte sureste del área de amortiguamiento del Parque Nacional Corcovado, Península de Osa. Costa Rica. Tesis de Maestría. Instituto Internacional en Conservación y Manejo de Vida Silvestre.

Ceballos, G., \& Oliva, G. 2005. Los Mamíferos de México. Fondo de Cultura Económica. CONABIO.

Chávez, C., Ceballos, G., Medellín, R. \& Zarza, H. 2007. Primer Censo Nacional del Jaguar, pp. 113 -141. In: Ceballos, G., C. Chávez, R. List, \& H. Zarza (Eds.). Conservación y Manejo del Jaguar en México: Estudios de Caso y Perspectivas. (CONABIO-Alianza WWF/Telcel-Universidad Nacional Autónoma de México, Distrito Federal, México.

Crawshaw, P. 1995. Comparative ecology of ocelot (Felis pardalis) and jaguar (Panthera onca) in a protected subtropical forest in Brazil y Argentina. Tesis de Doctorado. Universidad de Florida. Gainesville, Florida, USA.

De Almeida, A. T., Silveira, L. \& Felizola, J. A. 2004. Niche separation between the maned wolf (Chrysocyon brachyurus), the crab-eating fox (Dusicyon thous) and the hoary fox (Dusicyon vetulus) in central Brazil. Journal of Zoology, 262: 99-106.

Di Bitetti, M., Paviolo, A. \& De Angelo, C. 2006. Density, habitat use and activity patterns of ocelots (Leopardus pardalis) in the Atlantic Forest of Misiones, Argentina. Journal of Zoology, 270: 153163. 
Dillon, A. 2005. Ocelot density and home range in Belize, Central America: camera-trapping and radio telemetry. Master Thesis. Virginia Polytechnic Institute and State University.

Dillon, A. \& Kelly, M. J. 2008. Ocelot home range, overlap and density: comparing radiotelemetry with camera trapping. Journal of Zoology, 275:391-398.

Emmons, L. 1988. A field study of ocelots in Peru. Revue d'Ecologie de la Terre et de la Vie, 43: 133157.

Fragoso, J. M. 1988. Home range and movement patterns of White-Lipped Peccary (Tayassu pecari) herds in the Northern Brazilian Amazon. Biotropica, 30: 458-469.

Galindo-Leal, C. \& Lira, I. 2011a. Los Mamíferos de la Selva Zoque: Riqueza, pp. 211-221. In: D. Ortega del Valle, L. Carranza, \& J. Martínez-Pérez (Eds.) Una Mirada desde el Corazón de la Jícara de Oro (Experiencias de Conservación en la Selva Zoque de los Chimalapas). WWF-México. México, D.F.

Galindo-Leal, C. \& Lira, I. 2011b. Los Mamíferos de la Selva Zoque: Uso y Conservación, pp. 222235. In: D. Ortega del Valle, L. Carranza, \& J. Martínez-Pérez (Eds.) Una Mirada desde el Corazón de la Jicara de Oro (Experiencias de Conservación en la Selva Zoque de los Chimalapas). WWFMéxico. México, D.F.

García, E. 1973. Modificaciones al sistema de clasificación climática de Köppen. Instituto de Geografía, U.N.A.M. México.

Gobierno del Estado de Oaxaca. 1990. Tequio por Chimalapas. Comité Estatal de Planeación para el Desarrollo de Oaxaca, Subcomité Especial del COPLADE para la Microrregión de los Chimalapas, Vocalía Ejecutiva de los Chimalapas.

González, M. F. 2004. Las Comunidades Vegetales de México. Secretaría de Medio Ambiente y Recursos Naturales, Instituto Nacional de Ecología. 2a , ed., México, D.F.

Hall, E. R. 1981. The Mammals of North America. Vols. I, II. John Wiley y Sons. New York, EUA.

Haines, A., Janecka, J., Tewes, M., Grassman Jr., L. \& Morton, P. 2006. The importance of private lands for ocelots Leopardus pardalis in the United States. Oryx, 40: 1-5.

Harmsen, B., Foster, R., Silver, S., Ostro, L. \& Doncaster, P. 2010. Differential use of trails by forest mammals and the implications for camera-trap studies: a case study from Belize. Biotropica, 42: 126-133.

IUCN. 2011. IUCN Red List of Threatened Species. (Consultado en Mayo del 2011, www.iucnredlist. org).

Jenks, K. E., Chanteap, P., Damrongchainarong, K., Cutter, P., Cutter, P., Redford, T., Lynam, A. J., Howard, J., \& Leimgruber, P. 2011. Using relative abundance indices from camera-trapping to test wildlife conservation hypotheses - an example from Khao Yai National Park, Thailand. Tropical Conservation Science, 4: 113-131.

Krausman, P. 2002. Introduction to wildlife management. Prentice Hall, Nueva Jersey, EUU.

Lira-Torres, I., Naranjo, P. E., Güiris, A. D. \& Cruz, A. E. 2004. Ecología del Tapirus bairdii (Perissodactyla: Tapiridae) en la Reserva de La Biosfera "El Triunfo" (Polígono I), Chiapas, México. Acta Zoológica Mexicana. (n. s.), 20: 1-21.

Lira-Torres, I. 2006. Abundancia, Densidad, Preferencia de Hábitat y Uso Local de los Vertebrados en Tuza de Monroy, Santiago Jamiltepec, Oaxaca. Revista Mexicana de Mastozoología, 10: 6-31.

Lira-Torres, I. \& Briones-Salas, M. 2011. Impacto de la ganadería extensiva y cacería de subsistencia sobre la abundancia relativa de mamíferos en la Selva Zoque, Oaxaca, México. Therya, 2: 217244.

Lira-Torres. I., Galindo-Leal, C. \& Briones-Salas, M. 2012. Mamíferos de la Selva Zoque, México: Riqueza, Uso y Conservación. Revista de Biología Tropical (International Journal of Tropical Bio$\operatorname{logy),60:781-797.~}$ 
Lira-Torres, I. \& Ramos-Fernández, G. 2007. El Estado del Jaguar en los Chimalapas, Oaxaca, pp. 71-80. In: G. Ceballos, C. Chávez, R. List, \& H. Zarza (Eds.). Conservación y Manejo del Jaguar en México: Estudios de Caso y Perspectivas. Conabio-Alianza WWF/Telcel-Universidad Nacional Autónoma de México, México D.F.

Ludlow, M. E. \& Sunquist, M. E. 1987. Ecology and behavior of ocelots in Venezuela. National Geographic Research, 3: 447-461.

Maffei, L., Cuellar, E. \& Noss, J. 2002. Uso de trampas cámara para la evaluación de mamíferos en el ecotono Chaco-Chiquitanía. Revista boliviana de ecología y conservación ambiental, 11: 55-65.

Maffei, L., Cuellar, E. \& Noss, A. 2004. One thousand jaguars (Panthera onca) in Bolivia's Chaco? Camera trapping in the Kaa-Iya National Park. Journal of Zoology, 262: 295-304.

Maffei, L. \& Noss, A.J. 2008. How small is too small? camera trap survey areas and density estimates for ocelots in the Bolivian Chaco. Biotropica, 40: 71-75.

Maffei, L., Noss, A., Cuellar, E. \& Rumiz, D. 2005. Ocelot (Felis pardalis) population densities, activity and ranging behavior in the dry forests of eastern Bolivia: data from camera trapping. Journal of Tropical Ecology, 21: 1-6.

March, I. J. 1990. Evaluación del Hábitat y Situación Actual del Pecarí de Labios Blancos Tayassu pecari en México. Tesis de Maestría. Programa Regional en Manejo de Vida Silvestre para Mesoamérica y el Caribe. Universidad Nacional Heredia, Costa Rica.

Medellín, R. Azuara, D., Maffei, L., Zarza, H., Bárcenas, H., Cruz, E., Legaria, R., Lira, I. Ramos-Fernández, G. \& Ávila, S. 2006. Censos y Monitoreo, pp. 25-35. In: C. Chávez y G. Ceballos (Eds.). El Jaguar Mexicano en el Siglo XXI: Situación Actual y Manejo. CONABIO-ALIANZA WWF TELCEL-Universidad Nacional Autónoma de México. México. D. F.

Monroy-Vilchis, O., Rodríguez-Soto, C., Zarco-González, M. \& Urios, V. 2009. Cougar and jaguar habitat use and activity patterns in Central Mexico. Animal Biology, 59: 145-157.

Monroy-Vilchis, O., Zarco-González, M., Rodriguez-Soto, C., Soria-Díaz, L. \& Urios, V. 2011. Fototrampeo de mamíferos en la Sierra Nanchititla, México. Revista de Biología Tropical (International Journal of Tropical Biology), 59: 373-383.

Moreno, R., Kays, R., Giacalone, J., Aliaga-Rossel, E.\& Mares, R. 2006. Un estudio sobre la ecología del ocelote (Leopardus pardalis) en la isla de Barro Colorado, Panamá. Mesoamericana. X Congreso de la Sociedad Mesoamericana para la Biología y la Conservación, Ciudad Antigua, Guatemala.

Morruzzi, T. L., Fuller, T. K., DeGraaf, R. M., Brooks, R. T. \& Li, W. 2002. Assessing remotely triggered cameras from surveying carnivore distribution. Wildlife Society Bulletin, 30: 380-386.

Olguin Monroy, H., León, L., Samper-Palacios, U.M. \& Sánchez-Cordero, V. 2008. Mastofauna de la región de los Chimalapas, Oaxaca, México, pp 165-216. In: C. Lorenzo, E. Espinoza \& J. Ortega (Eds.). Avances en el estudio de los mamíferos II. Publicaciones Especiales, Vol. II, Asociación Mexicana de Mastozoología, A.C., México, D.F.

Ortiz Pérez, M. A., Hernández Santana, J. R. \& Figueroa, J.M. 2004. Reconocimiento Fisiográfico y Geomorfológico, pp. 43-54. In: A. J. García-Mendoza, M. J. Ordóñez \& M. Briones-Salas (Eds.). Biodiversidad de Oaxaca. Instituto de Biología, UNAM, Fondo Oaxaqueño para la Conservación de la Naturaleza-World Wildlife Fund, México.

Pina, G. P. L., Gómez, R. A. C. \& González, C. A. L. 2004. Distribution, habitat association and activity patterns of medium and large sized mammals of Sonora, Mexico. Natural Areas Journal, 24: 354-357.

Pinto de Sá Alves, L.C. \& Andriolo, A. 2005. Camera traps used on the mastofaunal survey of Araras Biological Reserve, IEF-RJ. Revista Brasileira de Zootecnia, 2: 231-246. 
Reid, A. F. 1997. A Field guide to the mammals of Central and Southeast Mexico. Oxford University Press, Nueva York.

Roberts, C. W., Pierce, B. L., Braden, A. W., Lopez, R. R., Silvy, N. J., Frank, P. A. \& Ranzom, D. 2006. Comparison of camera and road survey estimates for white-tailed deer. Journal of Wildlife Management, 70: 263-267.

Rovero, F. \& Marshall, A. 2009. Camera trapping photographic rate as an index of density in forest ungulates. Journal of Applied Ecology, 46: 1011-1017.

Rumiz, D., Fuentes, A., Rivero, K., Santibáñez, J., Cuellar, E., Miserendino, R., Fernández, I., Maffei, L. \& Taber, A. 2002. La biodiversidad de la Estancia San Miguelito, Santa Cruz-Bolivia: Una justificación para establecer reservas privadas de conservación. Instituto de Ecología, La Paz, Bolivia.

Rzedowsky, J. 1991. Vegetación de México. Limusa. México.

Sanderson, J. G. 2004. Protocolo para Monitoreo con Cámaras para Trampeo Fotográfico. Tropical Ecology Assessment and Monitoring (TEAM) Initiative. The Center for Applied Biodiversity Science (CABS). Conservación Internacional, USA.

Secretaría de Medio Ambiente, Recursos Naturales (SEMARNAT). 2010. Norma Oficial Mexicana NOM-059-ECOL-2010. Protección ambiental, especies de flora y fauna silvestres de México, categorías de riesgo y especificaciones para su inclusión, exclusión o cambio, y lista de especies en riesgo. Diario Oficial de la Federación, Jueves 30 de diciembre de 2010, 1: 1-77.

Silveira, L., Jácomoa, A. \& Diniz-Filhoa, J. 2003. Camera trap, line transect census and track surveys: a comparative evaluation. Biological Conservation, 114: 351-355.

Soisalo, M. K. \& Cavalcanti, S. M. C. 2006. Estimating the density of a jaguar population in the Brazilian Pantanal using camera-traps and capture-recapture sampling in combination with GPS radiotelemetry. Biological Conservation, 129: 487-496.

Srbek-Araujo, A.C. \& García, A. 2005. Is camera trapping an efficient method for surveying mammals in Neotropical forests? A case study in south-eastern Brazil. Journal of Tropical Ecology, 21: 121-125.

Sutherland, W. 1996. Ecological Census Techniques. Cambridge University, Cambridge, Gran Bretaña.

Tobler, M. W., Carrillo-Percastegui, S. E. \& Powell, G. 2009. Habitat use, activity patterns and use of mineral licks by five species of ungulate in South-Eastern Peru. Journal of Tropical Ecology, 25: 261-270.

Trolle, M. \& Kery, M. 2003. Estimation of ocelot density in the Pantanal using capture-recapture analysis of camera-trapping data. Journal of Mammalogy, 84: 607-614.

Trolle, M. \& Kery, M.. 2005. Camera-trap study of ocelot and other secretive mammals in the northern Pantanal. Mammalia, 69: 405-412.

Trolle, M. 2008. Brazilian tapir density in the Pantanal: a comparison of systematic camera-trapping and linetransect surveys. Biotropica, 40: 211-217.

Torres Colín, R. 2004. Tipos de Vegetación, pp. 105-117. In: A. J. García-Mendoza, M. J. Ordoñez \& M. Briones-Salas (Eds.). Biodiversidad de Oaxaca. Instituto de Biología, UNAM, Fondo Oaxaqueño para la Conservación de la Naturaleza, World Wildlife Fund, México.

Walker, S., Novaro, A. \& Nichols, J. 2000. Consideraciones para la estimación de abundancia de poblaciones de mamíferos. Mastozoología Neotropical, 7: 73-80.

Wallace, R. B., Gomez, H., Ayala, G. \& Espinoza, F. 2003. Camera trapping for jaguar (Panthera onca) in the Tuichi Valley, Bolivia. Mastozoología Neotropical, 10: 133-139.

Weckel, M., Giuliano, W. \& Silver, S. 2006. Jaguar (Panthera onca) feeding ecology: distribution of predator and prey through time and space. Journal of Zoology, 270: 25-30. 
Wendt, T. 1989. Las Selvas de Uxpanapa, Veracruz-Oaxaca, México: Evidencia de Refugios Florísticos Cenozoicos. Anales del Instituto de Biología. Serie Botánica, 58: 29-54.

Wilson, D. E., Rusell, F., Nichols, J. D., Rudran, R. \& Foster, M. S. (Eds.). 1996. Measuring and Monitoring Biological Diversity, Standar Methods for Mammals. Smithsonian Institution Press. Washington and London.

Yasuda, M. 2004. Monitoring diversity and abundance of mammals with camera traps: a case study on Mount Tsukuba, central Japan. Mammal Study, 29: 37-46. 